\title{
Molecular characterization of a cDNA encoding an excretory-secretory antigen from Toxocara canis second stage larvae and its application to the immunodiagnosis of human toxocariasis
}

\begin{abstract}
The cDNA encoding an excretoryï secretory antigen from the second stage larvae of Toxocara canis has been characterized. Sequence analysis revealed an open reading frame encoding a protein of 226 amino acid residues $(\mathrm{Mr}=24 \mathbb{1} 98)$. Sequence database searches showed similarities to regions corresponding to epidermal growth factor-like and lectin-like domains of the core proteins of vertebrate chondroitin sulfate proteoglycans, which are major components of the extracellular matrix. The T. canis core protein was expressed as a fusion protein with thioredoxin A using an Escherichia coli expression system, and then affinity purified on a metal affinity resin in the presence of $8 \mathrm{M}$ urea. When the purified recombinant T. canis protein was used as an antigen, immunoblot analysis revealed the protein specifically reacted with sera from toxocariasis patients. The antigenic protein did not react with sera from patients with Brugia malayi infection, dirofilariasis, or ascariasis. In some cases of anisakiasis, cross-reactions were observed; however, the cross-reacting bands disappeared when anisakiasis sera preabsorbed with Anisakis antigen were used, indicating that the recombinant $\mathrm{T}$. canis protein is very promising for use as an immunodiagnostic antigen for human toxocariasis.
\end{abstract}

Keyword: Excretory-secretory antigen; Human toxocariasis; Immunodiagnosis; Proteoglycan core protein; Recombinant antigen; Second stage larvae; Toxocara canis 